Check for updates

1 NHS Lothian

2 The George Institute for Global Health

3 Newcastle University

Cite this as: BMJ 2021;375:n2463

http://dx.doi.org/10.1136/bmj.n2463

Published: 11 October 2021

\title{
Trying to fit in: dressing like a doctor
}

\author{
Kate Womersley, ${ }^{1,2}$ Helen Hare, ${ }^{1}$ Stephanie BH Kelly, ${ }^{1}$ Eilidh Wilcockson ${ }^{3}$
}

After her objective structured clinical examination (OSCE) in July, Eilidh Wilcockson, a third year medical student at Newcastle University, received some surprising feedback: not about her clinical knowledge or examination technique, but about what she was wearing. She had been given a "professionalism yellow card," which is normally used to report violations of confidentiality, offensive language, or causing patient discomfort. In this case it was for the student's "short dress with no leg coverings." The "role player commented immediately after the station that it looked unprofessional," the notes on the card allegedly said. The examiner had added, "I agree."

The dress in question was dark navy with a subtle grey check. Sleeves fully covered the student's deltoids and axillae. The v neck's point reached the second intercostal space. Distally, the dress entirely hid the knees. The student had chosen flat shoes. She wore no jewellery and, as per NHS protocol, was bare below the elbows. Around $30 \mathrm{~cm}$ of calf was also bare between the tibial tuberosity and ankle. It's uncomfortable to describe a person's appearance with anatomical accuracy unless that's your job. It was the examiner's job to assess the student's clinical interaction with the patient, not to disparage her clothing choices.

When questioning the yellow card Wilcockson was told it was "the most inappropriate dress they had ever seen in their entire time of examining." After attempts to respond to the accusation through the university's formal complaints procedure, Wilcockson's friend tweeted the story with a photograph of the outfit.

"Have you attached the wrong photograph?" asked @Drbillgibson in response to the image, "That's of someone wearing pretty conservative business attire." @Drlyndarose agreed, thinking that "the picture was an example shown to the student of what they should have worn." "Is this one of those magic dresses that looks different to different people?” quipped @Kumaranism.

In the messages of solidarity, sartorial freedom seemed to align with seniority. "I am a consultant," wrote @Mariekeemonts, "never ever do I wear tights in summer. Never had any complaints."

@Flynnmarina described the outfit as "exactly the sort of thing I advise my trainees to wear for the part 3 MRCOG exam or to give a paper at a conference."

Junior doctors also shared their experiences. One had been criticised because a patient could see the change in gradient of colour in her tights when she sat down. One remembered "comments on my sleeveless shirt for an OSCE once. Was on SSRIs at the time and the combo of sleeves + anxiety made me incredibly hot. As a still overheated doctor I only wear sleeveless tops and I've never had a single comment or complaint." Another despaired that the takeaway from her negative interactions seemed to be "women are distracting, please wear a paper bag.”

Standards of professional dress for women have varied wildly throughout history. The requirements for women doctors are more erratic than other professions. In the late 1960 , female doctors would be sent home to change if they arrived on the wards wearing trousers. Commenting on the photograph of Wilcockson, one doctor pointed out that "military dress uniform skirts are almost exactly that length and have been for many years. I'd suggest if it was long enough for the bastion of conservatism that was Sandhurst in 1990 it's long enough for an OSCE."

The appropriateness of appearance is also context specific: if you're the parent of a baby, vomit on the shoulder of your shirt is surely a forgivable imperfection; if you're hungover, it's almost certainly not; if you've just come from assisting a nauseated patient in the corridor, the stain is a manifestation of your professional duty.

People who are not cis, slim, straight, white, and male stand a greater chance of having their attire labelled "inappropriate." Several men reported that they had done their OSCEs in shorts without formal criticism. Women's "flaws," including their busts, bottoms, sweat, hips, wet hair, dyed hair, big hair, bra straps, ankles, shoulders, arms, nails, earrings, skin, or makeup can, and will, be called out. The policing of certain bodies smacks of high school uniform requirements to prevent girls from "distracting the boys." But it is our professional duty to be comfortable with bodies of all shapes, sizes, sexes, and altered by choice, pregnancy, lifestyle, disease, or surgery.

Many have asked where the harm is in setting standards for women's dress if they still pass the exam, ace the presentation, or secure the promotion. These incidents cause immediate as well as lasting distress. The daily work of trying to conform to outdated, discriminatory, and unpredictable "standards" is tiring. To source clothing which is not too loose or tight, not too flamboyant, with a neckline that is high enough but sufficiently feminine (the list goes on) takes time, money, and mental energy. After preparing for and delivering another gruelling audition for this profession, a comment about your appearance is a jarring humiliation. Each time the effort is judged insufficient, and dress codes are enforced by the whim of a person in power, access to the profession narrows.

These conflicts are not unique to the UK. In North American residency interviews, conversations between hopefuls often turn to whether wearing trousers instead of a skirt, or flat shoes rather than 
heels, is worth the "risk." These risks seem greater in male dominated fields. Obligatory heels for surgical interviews should be a flattering height, but not too "sexy." For a profession that prides itself on practical and often physical work, these requirements feel particularly out of place. The consequence of missing the mark can mean a person's future in a specialty is jeopardised. US medical schools retain incidents of unprofessionalism in performance records which are submitted with training programme applications. Should a professionalism violation be upheld, details will be distilled over time, leaving a permanent mark on a student's record.

The NHS's current unwritten dress code isn't working. The details need to be guessed by doctors while sizing up the prejudices of their seniors and erasing their own social, cultural, religious, and economic backgrounds. Of course, there are inappropriate ways to be dressed as a clinician. But no single outfit will satisfy the opinions of every one of our patients.

Might a uniform simplify things? Perhaps, but it would have to be inclusive, debated, published, and reviewed. If "leg coverings" were enforced, there would at least be an opportunity for coordinated outcry. During the pandemic, the routine wearing of scrubs simplified aspects of daily decision making. If they continue to be acceptable for all, staff deserve scrubs that comfortably fit and protect a variety of bodies. Currently, these glorified pyjamas masquerade as being "unisex," but are designed for slim men.

The question of dress is connected to many other problems in a culture of professional sexism. A BMA report published last month found that nine out of 10 female doctors in the UK have experienced sexism at work. The "failure to tackle structures and policies that favour a workforce of men, allowing sexist attitudes and gender bias to remain prevalent has led to unequal opportunities for women and is causing problems in their day-to-day working lives," the authors conclude. Why is this only coming to light now? Why haven't women said anything? They have-daily, on and offline. But complaint processes are time consuming, emotionally fraught, and rarely lead to satisfactory outcomes. Speaking up can also prompt further questions about professionalism. In this case, Newcastle University responded by apologising "for any offence or distress caused by this incident.”

"All comments made by patients in exams are passed on to students as feedback," the university said. Would the university have passed on racist, homophobic, or other discriminatory comments to a student? The minimum professional obligation of a clinician, or examiner, is to filter out tangential personal opinion, even if they happen to agree with it.

Criticisms of a colleague's appearance are unacceptable-that is unless the critic is prepared to forensically detail the infringement in writing and defend it formally. If the transgression is not egregious or part of a long term pattern, it is surely right not to comment.

Part of being a professional is protecting colleagues from these kinds of accusations, contributing to a working environment that respects the humanity and dignity of everyone. Professionalism is about values, not hemlines. 